\title{
Un análisis sobre percepciones sociales de la ciudadanía. La normalización de la prostitución como servicio necesario
}

\author{
An analysis of social perceptions of citizenship. The \\ normalization of the prostitution of women as a necessary
}

service

\author{
Esther Torrado Martín-Palomino \\ Yasmina Romero Morales \\ Josué Gutiérrez Barroso
}

Recibido: 02/10/2017

Aceptado: 12/09/2018

\section{RESUMEN}

Los datos reflejados en este artículo son parte del Estudio sobre la prostitución de mujeres en Canarias, financiado por el Instituto Canario de Igualdad y realizado por la Universidad de La Laguna. Se trata de un proyecto de investigación que ha analizado las opiniones de las mujeres en prostitución, los demandantes, los profesionales que intervienen directa o indirectamente en el fenómeno y las percepciones de la ciudadanía. La información aquí reflejada procede de los resultados de la encuesta realizada a personas residentes en Canarias para conocer sus percepciones y opiniones sobre la prostitución de mujeres.

Palabras clave: percepción, prostitución, mujeres, ciudadanía

\section{ABSTRACT}

The data reflected in this article are part of the Study about Women Prostitution in Canary Islands, funded by the Canary Islands Equality Institute and carried out by the University of La Laguna. It is a research project that has analyzed the

Esther Torrado Martín-Palomino es doctora en Sociología. Profesora investigadora del Departamento de Sociología y Antropología de La Laguna, miembro del Instituto Universitario de Estudios de las Mujeres (IUEM). Correo electrónico: estorra@ull.edu.es. ID: https://orcid.org/0000-0002-9144-5616

Yasmina Romero Morales es doctora en Estudios Filológicos y licenciada en Filología por la Universidad de La Laguna. Diploma de Estudios Avanzados en Estudios Árabes e Islámicos. Máster en Estudios Feministas, Violencia de Género y Políticas de Igualdad. Correo electrónico: yromerom@ull.edu.es. ID: https://orcid.org/0000-0003-0255-5782

Josué Gutiérrez Barroso es doctor en Sociología y licenciado en Sociología por la Universidad de La Laguna. Profesor del Departamento de Sociología y Antropología de la Universidad de La Laguna y de la Escuela Universitaria de Turismo Iriarte. Correo electrónico: jgutierb@ull.edu.es. ID: https://orcid.org/0000-0001-5968-3254

Cómo citar este artículo: Torrado Martín-Palomino, B.; Romero Morales, Y. y Gutiérrez Barroso, J. (2018). Un análisis sobre percepciones sociales de la ciudadanía. La normalización de la prostitución de mujeres como un servicio necesario. Atlánticas. Revista Internacional de Estudios Feministas, 3 (1), 164-174 doi: http://dx.doi.org/10.17979/arief.2018.3.1.3078 
opinions of women in prostitution, the plaintiffs, the professionals who intervene directly or indirectly in the phenomenon and the perceptions of citizenship. The information reflected here comes from the results of the survey conducted on people living in the Canary Islands to know their perceptions and opinions about the prostitution of women.

Keywords: perception, prostitution, women, citizenship 


\section{OBJETIVO DEL ESTUDIO Y METODOLOGÍA DE LA INVESTIGACIÓN ${ }^{1}$}

El objetivo general del estudio era conocer la prostitución de mujeres en Canarias. La fase llevada a cabo durante el año 2017 se centró en conocer en profundidad la prostitución de mujeres a través de los discursos y perfiles de los demandantes y de la percepción de la ciudanía canaria. En este artículo nos centraremos en el objetivo específico correspondiente a analizar la percepción y discursos públicos de esta ciudadanía mayor de 18 años y en algunos indicadores analizados a través de una encuesta telefónica llevaba a cabo en las siete islas del archipiélago para conocer la imagen y posición con respecto a la prostitución.

En el año 2016 se realizó la Primera Fase del estudio sobre la prostitución de mujeres en Canarias, con entrevistas en profundidad a mujeres prostituidas y Focus Group con profesionales que intervenían directa o indirectamente con estas mujeres. Durante el año 2017, se realizó la Segunda Fase del estudio con entrevistas en profundidad, en este caso, a hombres consumidores de prostitución y una encuesta telefónica a la población de las siete islas para conocer las percepciones sobre la prostitución de mujeres².

En este artículo nos vamos a centrar en esta última técnica, la encuesta telefónica y sus resultados más relevantes. La recogida de datos se realizó mediante el sistema CATI (encuestas telefónicas asistidas por ordenador, en sus siglas en inglés) a través de una plataforma diseñada por Servicios Estadísticos de Canarias específicamente para esta encuesta. Asimismo, se realizó a una muestra representativa de personas residentes en Canarias mayores de 18 años, mediante el muestreo aleatorio simple ${ }^{3}$.

El trabajo de campo, a su vez, se desarrolló en los meses de septiembre y octubre y participaron un total de 1.212 personas, con un error típico $+/-2,81 \%$ para un nivel de confianza del 95\% y $p=q=0,5$. El cuestionario tenía 16

\footnotetext{
${ }^{1}$ Este trabajo forma parte del Proyecto "Percepción Social de la Prostitución de Mujeres y análisis de la demanda prostitucional en Canarias 2017. La normalización de la violencia sexual contra las mujeres como un servicio" financiado por el Instituto Canario de Igualdad del Gobierno de Canarias y encargado a la Universidad de La Laguna.

${ }^{2}$ La encuesta telefónica durante los meses de septiembre y octubre del 2017 fue ejecutada, codificada, grabada y depurada por los Servicios Estadísticos de Canarias.

${ }^{3}$ La población canaria asciende, actualmente, a 1.742 .605 personas, de las cuales 858.427 son hombres y 884.178 son mujeres.
} 
preguntas, y tras la introducción del estudio y la invitación a colaborar, se planteó una cuestión introductoria sobre las preocupaciones generales que los entrevistados consideraban importantes y que afectaban directamente a la comunidad autónoma de Canarias. A partir de esta pregunta preliminar, se inició el cuestionario propiamente dicho; en primer lugar, con preguntas relacionadas con la caracterización sociodemográfica de la población entrevistada y, en segundo lugar, con preguntas relativas a su percepción sobre la prostitución. Respecto a este bloque, eje central de nuestro estudio, se indagó sobre el posicionamiento - a favor o en contra - que le merecía la regulaciónlegalización de la prostitución. Posteriormente, sobre quiénes consideraban que eran los principales beneficiarios del fenómeno, también sobre las víctimas y los consumidores de prostitución e, incluso, si conocían a alguno de estos consumidores y qué opinión les merecía.

\section{POBLACIÓN Y MUESTRA}

La población objetivo de estudio estuvo formada por personas mayores de edad de la comunidad autónoma de Canarias. Los datos poblacionales utilizados procedieron del padrón municipal de habitantes de Canarias a fecha 01/01/2016 publicados por el Instituto Canario de Estadística (ISTAC).

La muestra de mujeres y hombres mayores de 18 años residentes en Canarias que respondió a esta encuesta estuvo formada, según sexos, por un 57\% de mujeres y un $42 \%$ de hombres. Según la isla de residencia, el $79 \%$ de la muestra residía en las islas capitalinas - Tenerife o Gran Canaria-, el resto de participantes se distribuyó entre las demás islas. En lo tocante a la edad, el 31\% de la población tenía 61 o más años; el 24\% tenía entre 51 y 60 años y el 31\% tenía entre 41 y 50 años. Sólo el 10\% tenía menos de 30 años.

Por lo que respecta a la situación laboral de las personas encuestadas residentes en Canarias, el $43 \%$ de la población estaba ocupada en el momento de la realización de la encuesta, mientras que el 17\% se encontraba en situación de desempleo. El 25\% de la población, a su vez, se hallaba jubilada, el 9\% se declaraba como ama/o de casa y el 4,6\% estudiante. En el 59\% de la muestra de mujeres y hombres, su estado civil era casados o con pareja, mientras que el $40 \%$ se encontraba en otra situación civil diferente, esto es, solteros/as, divorciados/as, separados/as y viudos/as. El 73\% de la población encuestada declaró que tenía hijos/as y el 26,9\%, por su parte, que no los tenía. 


\section{INTERPRETACIÓN DE DATOS Y RESULTADOS SOBRE LA PERCEPCIÓN SOCIAL DE LA PROSTITUCIÓN EN CANARIAS}

A continuación, se detallan algunos de los resultados extraídos de esta encuesta telefónica, donde sólo 1 de cada 4 personas — tanto hombres como mujeresconsideraban que la prostitución debería prohibirse, frente a 3 de cada 4 que opinó lo contrario (77\%), no apreciándose diferencias según el sexo.

Sin embargo, si se tiene en cuenta el estado civil y el sexo de la población encuestada (gráfico 1), se deduce que las personas casadas o en pareja consideraban que la prostitución debería abolirse o prohibirse, en mayor medida que las solteras, divorciadas, separadas o viudas (un $25 \%$ frente a un $20 \%$ ). Los resultados también indican, en comparación con las personas que viven en pareja o están casadas, que aquellas que viven solas tienen un discurso asociado a una mayor tolerancia hacia la prostitución.

Gráfico 1. Opinión de mujeres y hombres sobre la prohibición de la prostitución según su estado civil y sexo del encuestado/a. 2017. (\%)

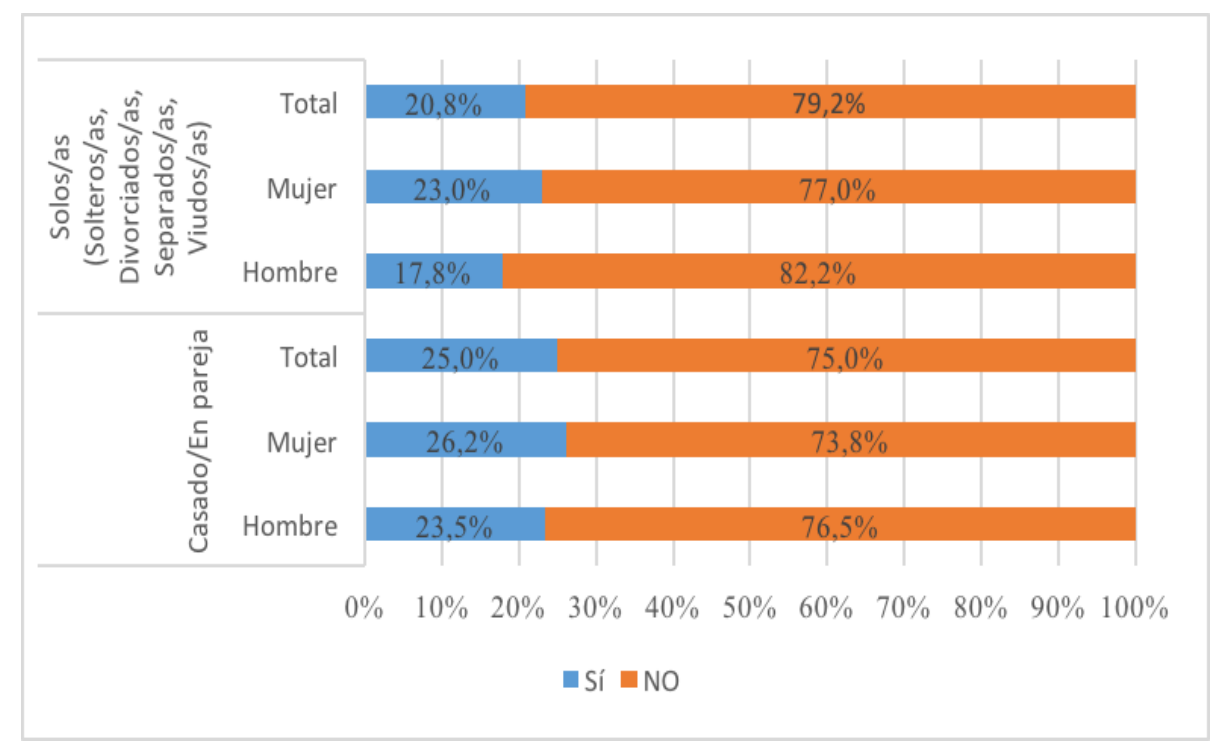

Fuente: Elaboración propia a partir de los datos de la encuesta

En general, el 23\% de población encuestada opinaba que debería prohibirse la prostitución. De esas personas que afirmaron que debería prohibirse, y tal como se muestra en el gráfico 2 , el $38 \%$ destaca que convendría castigar tanto al proxeneta como a la persona que ejerce la prostitución y a la "clientela" ${ }^{4}$. Sin

\footnotetext{
4 En este estudio, cuando se nombra la palabra "cliente" o "clientela" y demás conceptos similares, aparecerá entrecomillado, pues es terminología utilizada por las personas que han colaborado con este estudio.
} 
embargo, el 32\% afirmó que sólo debería castigarse al proxeneta, el 11\% a los "clientes" y el 3\% a la persona que ejerce la prostitución. El 9\% declaró que sólo debería castigarse al "cliente" y al proxeneta.

Gráfico 2. A quién habría que castigar si se prohibiera la prostitución. 2017. (\%)

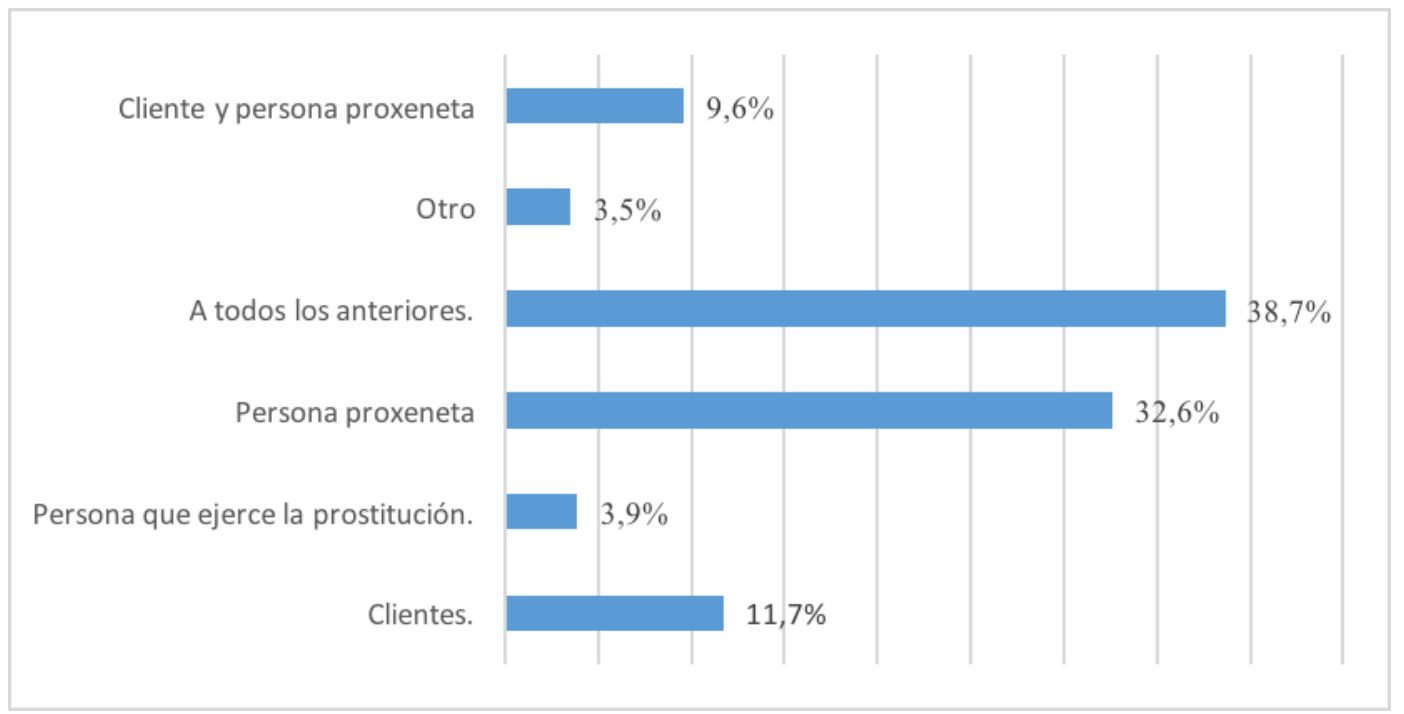

Fuente: Elaboración propia a partir de los datos de la encuesta

En ese sentido, la población que se posicionaba a favor de prohibir la prostitución consideró, de modo mayoritario, que se debería penalizar a proxenetas y a las personas consumidoras de la prostitución en mayor medida que a las personas que ejercen la prostitución.

\subsection{Percepción sobre el perfil de los hombres que demandan prostitución}

Otro de los datos que aportó la encuesta fue la percepción que la población poseía sobre el perfil de las personas consumidoras de prostitución (gráfico 3). En primer lugar, casi la mitad de las personas encuestadas, tanto mujeres como hombres, creía que los demandantes de prostitución eran personas casadas o en pareja (47\%). Sorprendentemente, el $28 \%$ de la población encuestada declaró, en primer lugar, que tanto los hombres como las mujeres eran consumidores por igual de prostitución. En segundo lugar, más de la mitad de las personas encuestadas opinaron que la prostitución era consumida por hombres solteros (51\%) y, en tercer lugar, por hombres jóvenes (52\%).

Por tanto, puede concluirse que la población percibe que las personas consumidoras de prostitución son principalmente hombres casados o en pareja en un primer lugar; hombres solteros en un segundo lugar; y hombres jóvenes 
en último lugar, opinión que es sostenida prácticamente por igual tanto en mujeres como en hombres.

Llama de manera especial la atención, el alto porcentaje de hombres y mujeres que piensan que, incluso en primer lugar, la "clientela" son hombres y mujeres por igual.

Gráfico 3. Opinión de la población encuestada sobre cuál el perfil de las personas consumidoras de prostitución según orden de preferencia. 2017. (\%).

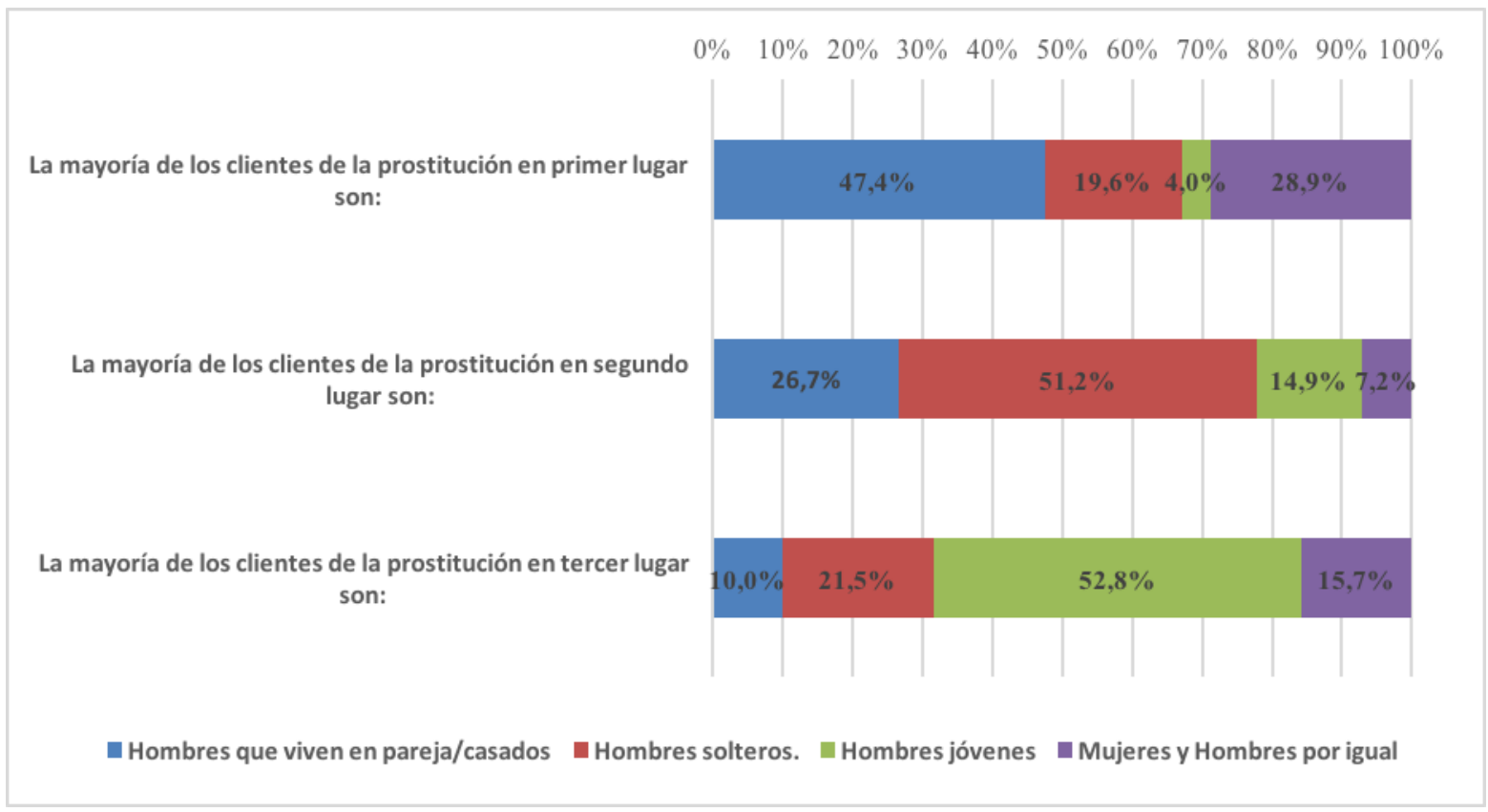

Fuente: Elaboración propia a partir de los datos de la encuesta

\subsection{Opinión de la ciudadanía sobre el perfil y motivaciones de los hombres para consumir prostitución}

En el gráfico 4 se muestra la opinión del tipo de hombre que consume prostitución. La mayoría de las personas encuestadas (91\%) consideraron que aquellas que consumían prostitución eran hombres que necesitan satisfacer sus necesidades sexuales en primer lugar, hombres con matrimonios frustrados en segundo lugar (83\%) y hombres solos en tercer lugar $(80 \%)$.

Además, 3 de cada 4 personas opinaron que los hombres consumidores de prostitución eran, también y mayoritariamente, consumidores de pornografía (74\%) y hombres con patologías mentales a los que les gustan mucho las mujeres (70\%). Sin embargo, más de la mitad de la población encuestada tanto de hombres como de mujeres, consideró que los consumidores de prostitución de mujeres eran mayoritariamente hombres "normales" (67\%), hombres que 
viven alejados de sus familias (65\%) y "hombres muy hombres" (64\%).

Gráfico 4. Opinión de la población encuestada sobre las razones por las que se consume prostitución. 2017 (\%)

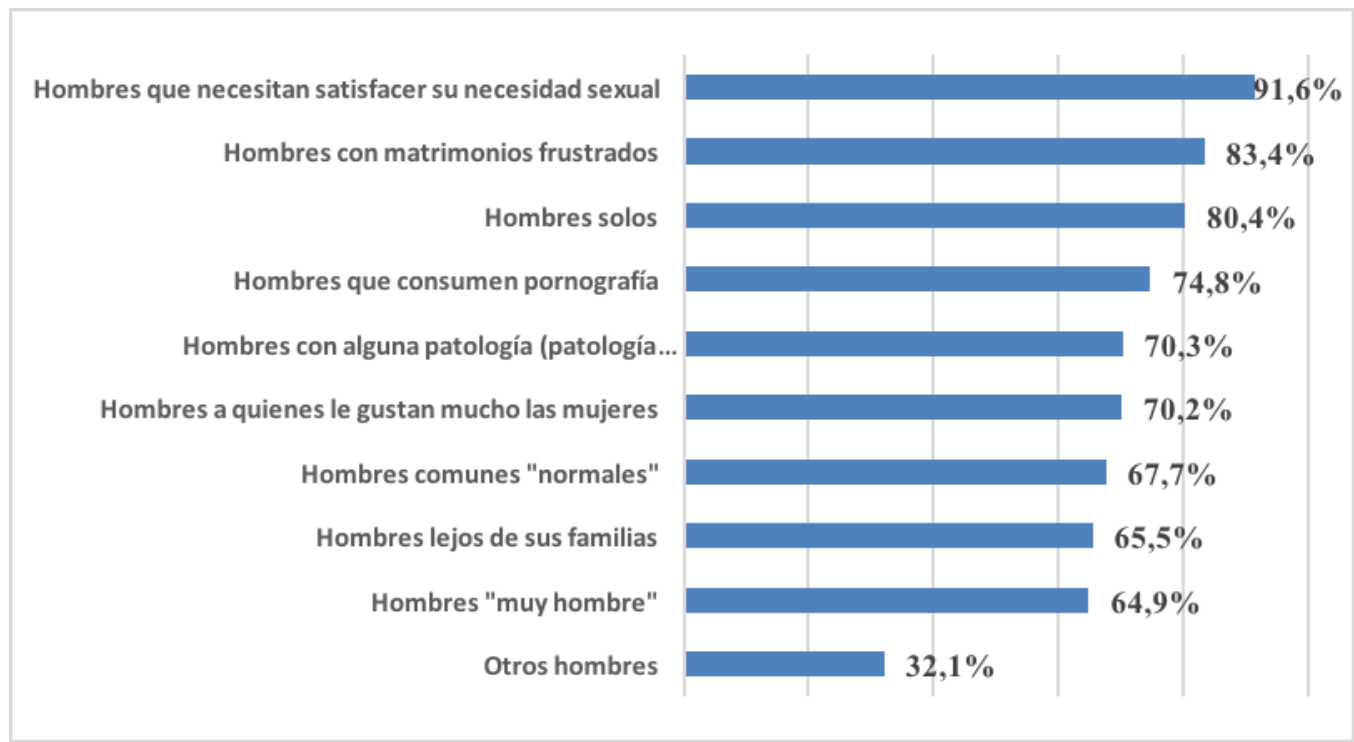

Fuente: Elaboración propia a partir de los datos de la encuesta

En definitiva, parece que la mayoría de la población canaria encuestada se inclina por pensar, en primer lugar, que las personas que consumen prostitución tienen motivaciones personales e íntimas. Sólo como segunda opción, pueden llegar a pensar que estas razones están asociadas a conductas negativas como el consumo de pornografía e, incluso, a patologías psicológicas. Sin lugar a dudas, las opiniones más señaladas desvelan los tópicos y estereotipos existentes que comportan cierta normalización de la prostitución.

Resulta conveniente el análisis de esos tipos de hombres consumidores de prostitución según el sexo de la persona encuestada (gráfico 5). La mayoría de mujeres y hombres consideran que las personas consumidoras de prostitución son principalmente hombres que necesitan satisfacer sus apetitos sexuales (más del $90 \%$ ), tienen matrimonios frustrados (más del $80 \%$ ) o se trata de hombres que están solos (alrededor del 80\%). A su vez, 3 de cada 4 mujeres consideran que son consumidores de pornografía (frente a tan solo el $72 \%$ de los hombres) y el $74 \%$ de las mujeres, consideran que se trata de hombres con patologías psicológicas (frente al 66\% de los hombres). 
Gráfico 5. Opinión de la población encuestada sobre las razones por las que se consume prostitución según sexo del encuestado/a. 2017. (\%)

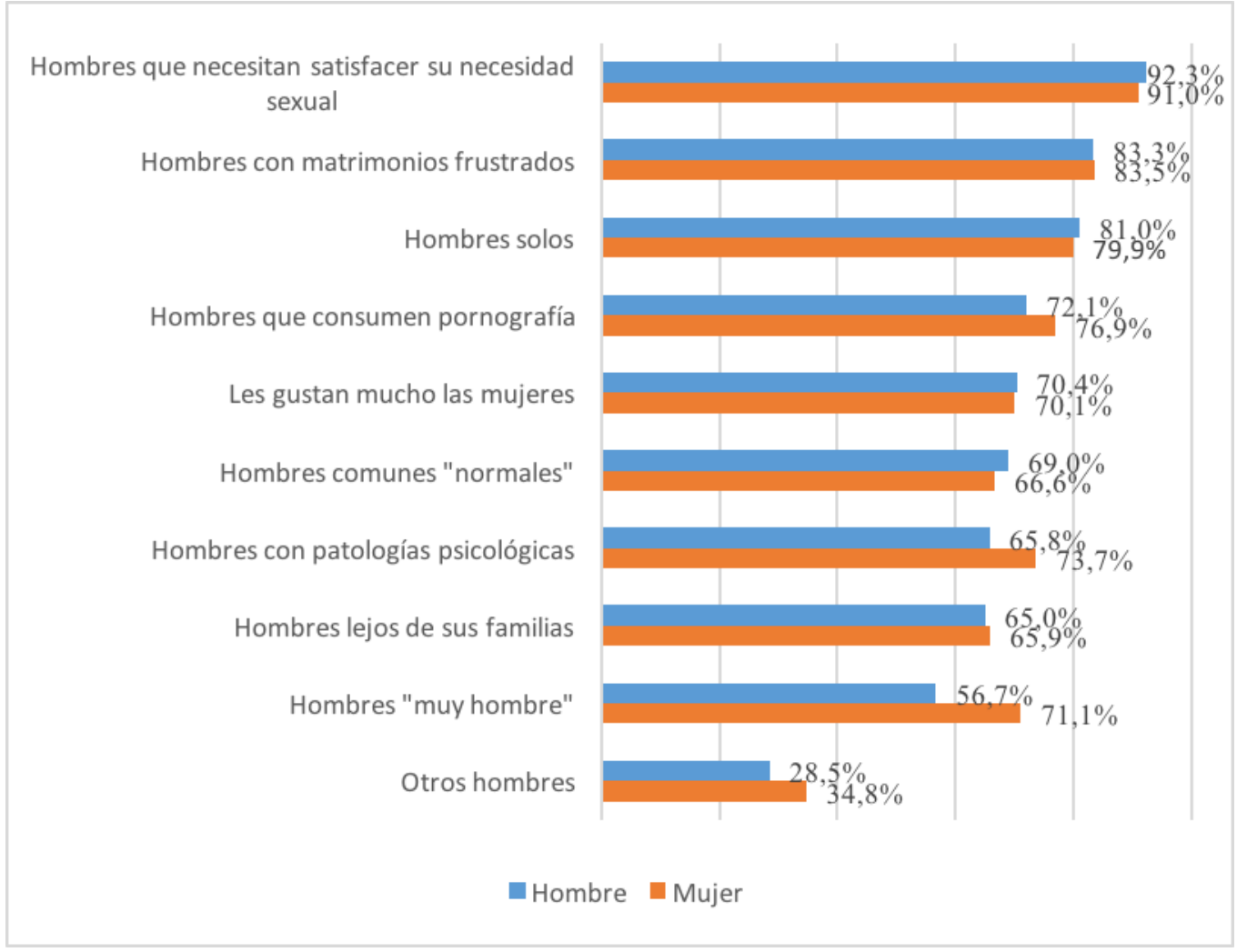

Fuente: Elaboración propia a partir de los datos de la encuesta

Estas dos categorías son las únicas donde, a tenor de los datos recogidos, las mujeres superan a los hombres calificando negativamente a los demandantes de prostitución. Las personas encuestadas, también opinan que se trata de hombres a los que les gustan mucho las mujeres (70\% aproximadamente de los hombres y de las mujeres), hombres "normales" (69\% de los hombres y 67\% de las mujeres respectivamente), hombres "lejos de sus familias" (66\%) y "muy hombres" (57\% de los hombres y 71\% de las mujeres). También destaca el gran porcentaje de mujeres y hombres que no caracterizan a esa "clientela" con ninguna de estas tipologías y ofrece otras explicaciones $(28,5 \%$ de los hombres y el $35 \%$ de las mujeres).

Esto, principalmente, revela que la opinión de la población respecto a la prostitución de mujeres está llena de mitos y estereotipos, no teniendo una opinión clara de quiénes forman parte de esa "clientela potencial demandante de prostitución de mujeres", ni de sus motivaciones para hacerlo, más allá de las expresiones formales y tópicas que se les había ofrecido en nuestra encuesta como respuestas estándares. 


\section{CONCLUSIONES}

La encuesta en términos globales refleja que la normalización de la prostitución en Canarias es un hecho, a pesar de que la actividad no sólo está ligada a la feminización y a la posición vulnerable de las mujeres, sino a mitos que contribuyen a consolidar esa desigualdad y la violencia de género que conlleva. A pesar de que en la Primera Fase del estudio de 2016 se pudo apreciar que el origen de las mujeres en situación de prostitución estaba íntimamente ligado a la vulnerabilidad y a la procedencia de sociedades patriarcales $y$, también, pese a que se ha demostrado que la prostitución es una oportunidad de lucro cuya plusvalía deriva de la vulneración de los derechos humanos, existen numerosas resistencias culturales para identificar la prostitución con la violencia.

Quizás, esta negación deriva de la banalización y normalización social de una actividad, donde sus responsables son eximidos de cualquier consecuencia ética, como hemos visto en los resultados de la encuesta. Se obvia el negocio internacional que ha generado el asentamiento de una red de redes que surten el mercado prostitucional $\mathrm{y}$, a pesar de que es una actividad mayoritariamente feminizada, en casi ningún caso se reconoce que en este sistema las mujeres no ejercen ningún tipo de dominio. Por ende, no se traen a colación las verdaderas condiciones de explotación y violencia en la que intentan sobrevivir.

La consideración de esta actividad como una mera transacción económica, derivada de servicios que ofrecen personas bajo condiciones de igualdad y libertad es una falacia que omite el dolor, el reconocimiento de la condición de víctima y las necesarias medidas de reparación social. Diversas ideas-fuerza en forma de mitos, han tratado de justificar estas violencias intrínsecas al sistema prostitucional, mediante narrativas desvirtuadas y falsas ideas acerca de un sexo de centralidad masculina, alimentado por la idea del poder y el control sobre las mujeres. Así, estos mitos transmitidos desde la tradición patriarcal, han contribuido históricamente a justificar las violencias sexuales contra las mujeres, desde entramados imbricados en la fuerza o en tesis biologicistas sobre una naturaleza diferencial y superior por parte de los hombres. Así, observamos que una de las razones principales que esgrimen las personas encuestadas sobre las razones por las que los hombres demandan prostitución es hacer alusión a las necesidades sexuales, el no vivir en pareja o la soledad. 
Finalmente y, en consecuencia, la transmisión cultural de falsas creencias y tradiciones, cobra verdadero sentido de funcionalidad, al justificar y sostener un andamiaje patriarcal y generizado como servicio necesario, aunque la mayoría de las personas en prostitución sean mujeres en situación de violencia y vulnerabilidad. En ese sentido, y bajo la conjunción de estas ideas, la ciudadanía pierde su capacidad de reaccionar y de percibir situaciones bajo sus verdaderos significados, entendiendo falsamente la prostitución como algo necesario y anulando la validez de las narrativas de las víctimas. 